\title{
Approximation of certain multivariate integrals
}

\author{
Jane M. Olson and Lisa A. Weissfeld \\ Department of Biostatistics, Unitersity of Michigan, Ann Arhor, MI 48109, USA
}

Received March 1990

Revised May 1990

\begin{abstract}
A Taylor series approximation to multivariate integrals taken with respect to a multivariate probability distribution is proposed and applied to the computation of multivariate normal probabilities and conditional expectations. The approximation does not require that the multivariate distribution have a structured covariance matrix and, in its simplest form, can be written as the product of univariate integrals. The approximation is compared to that of Mendell and Elston (1974) for computing bivariate normal probabilities.
\end{abstract}

Keywords: Multivariate normal probabilities, Taylor series.

\section{Introduction}

Computation of some multivariate probabilities and expectations, most notably the multivariate normal, require evaluation of multivariate integrals. For many applications, including simulation studies and iterative procedures, a computationally simple approximation is desired. Although a large number of approximations to multivariate integrals have been proposed, many are cumbersome or make assumptions which limit their application. (See, for example, Johnson and Kotz, 1972, for an summary of some of these methods.) The present work was motivated by the need to simplify computation of the multivariate integrals required to obtain expectations taken with respect to the multivariate normal distribution, as well as to obtain multivariate normal probabilities. However, the proposed method is general enough to be useful for other multivariate distributions and is therefore presented for the general case.

We propose a Taylor series approximation which reduces the multivariate integral to functions of univariate integrals and show how the method can be used to approximate multivariate probabilities and integrals taken with respect to a multivariate density (Section 2). In Section 3, the method, which does not require a structured covariance matrix, is used to approximate multivariate normal probabilities. In its simplest form, the approximation is similar to the approximation of Mendell and Elston (1974) and the latter method is described. Also in Section 3, the accuracy of the approximation in evaluating multivariate normal tail probabilities is examined and, in the bivariate case, compared to that of the Mendell-Elston approximation. In Section 4, the approximation is applied to a censored data situation which requires evaluation of conditional bivariate normal expectations.

\section{Method}

The Taylor series expansion is a well-known too for approximating the expected value of a function $g$ of a random variablc. Let $X$ bc a random variable with mean $\theta$. A convenient form for this expansion, after 
taking expectations, is

$$
E[g(X)]=g(\theta)+\frac{1}{2} g^{(2)}(\theta) E(X-\theta)^{2}+\frac{1}{6} g^{(3)}(\theta) E(X-\theta)^{3}+\cdots,
$$

where $g^{(2)}(\cdot)$ and $g^{(3)}(\cdot)$ are the second and third derivatives of $g(\cdot)$, respectively, and $E(\cdot)$ is the expectation operator. The function $g(x)$ is assumed to be continuous and to possess all derivatives at $x=\theta$. It is further assumed that a valid (i.e., convergent) Taylor series can be obtained.

We first demonstrate the principle of the method using an arbitrary trivariate distribution. Let $\boldsymbol{y}=\left(y_{1}, y_{2}, y_{3}\right)^{\mathrm{T}}$ be an observation from a trivariate distribution with joint density $f(\boldsymbol{y})$. Suppose we wish to determine the value of an integral $T[h(y)]$ taken with respect to the distribution $f(y)$ over a rectangular region $R=R_{1} \times R_{2} \times R_{3}$ :

$$
T[h(y)]=\int_{R} h(y) f(y) \mathrm{d} \boldsymbol{y}
$$

Multiplying the numerator and denominator of equation (2) by $\int_{R_{3}} f\left(y_{3}\right) \mathrm{d} y_{3}$, this expression can be written as

$$
\begin{aligned}
T[h(y)] & =\frac{\int_{R_{3}} f\left(y_{3}\right) \int_{R_{1} \times R_{2}} h\left(y_{1}, y_{2}, y_{3}\right) f\left(y_{1}, y_{2} \mid y_{3}\right) \mathrm{d} y_{1} \mathrm{~d} y_{2} \mathrm{~d} y_{3}}{\int_{R_{3}} f\left(y_{3}\right) \mathrm{d} y_{3}} \cdot \int_{R_{3}} f\left(y_{3}\right) \mathrm{d} y_{3} \\
& =E_{Y_{3}}\left(\int_{R_{1} \times R_{2}} h\left(y_{1}, y_{2}, Y_{3}\right) f\left(y_{1}, y_{2} \mid Y_{3}\right) \mathrm{d} y_{1} \mathrm{~d} y_{2} \mid Y_{3} \in R_{3}\right) \cdot \int_{R_{3}} f\left(y_{3}\right) \mathrm{d} y_{3},
\end{aligned}
$$

where $f\left(y_{1}, y_{2} \mid Y_{3}\right)$ is the density of $\left(y_{1}, y_{2}\right)^{\mathrm{T}}$ conditional on $Y_{3}$, and $E_{\mathrm{Y}_{3}}$ denotes the expectation with respect to $Y_{3}$. Using equation (1), we can now expand the conditional expectation in equation (3) in a Taylor series about $\theta=E_{Y_{3}}\left(Y_{3} \mid Y_{3} \in R_{3}\right)=E_{3}$, say. For simplicity, only the first term $g\left(E_{3}\right)$ will be used for the approximation; here

$$
E_{3}=\int_{R_{3}} y_{3} f\left(y_{3}\right) \mathrm{d} y_{3} / \int_{R_{3}} f\left(y_{3}\right) \mathrm{d} y_{3}
$$

is the marginal expectation of $Y_{3}$, given that $Y_{3} \in R_{3}$, and

$$
g\left(E_{3}\right)=\int_{R_{2}} f\left(y_{2} \mid E_{3}\right) \int_{R_{1}} h\left(y_{1}, y_{2}, E_{3}\right) f\left(y_{1} \mid y_{2}, E_{3}\right) \mathrm{d} y_{1} \mathrm{~d} y_{2} .
$$

Repeating the above process yields the first-order approximation

$$
T[h(y)] \simeq \int_{R_{1}} h\left(y_{1}, E_{2 \mid 3}, E_{3}\right) f\left(y_{1} \mid E_{2 \mid 3}, E_{3}\right) \mathrm{d} y_{1} \cdot \int_{R_{2}} f\left(y_{2} \mid E_{3}\right) \mathrm{d} y_{2} \cdot \int_{R_{3}} f\left(y_{3}\right) \mathrm{d} y_{3} .
$$

A similar sequence of steps can be used to approximate integrals of order higher than 3. Approximation (4) is easy to express and its computation requires the evaluation of only univariate integrals. The accuracy of the approximation can be improved by adding more terms to one or more of the Taylor series expansions; however, for higher dimensional integrals, the resulting expression may be unwieldy. 


\section{Approximation of multivariate normal probabilities}

\subsection{Multivariate normal probabilities}

The first-order Taylor series (TS) approximation to trivariate normal probabilities can be expressed as a special case of (4) by taking $h(y)=1$ and $f(y)$ as the trivariate normal density function. Approximate upper tail probabilities were computed using (4) for a standard trivariate normal distribution and are presented in Table 1. The IMSL subroutine MDNORD was used to compute normal probabilities. In the case of equal correlations and equal lower bounds (i.e., $\rho_{12}=\rho_{13}=\rho_{23}$ and $y_{1}=y_{2}=y_{3}$ ), the TS values are compared to table values (Kres, 1983); in all other cases, the TS values are compared to values computed using the FORTRAN program MULNORS (Schervish, 1984). Computation of the TS values required about $\frac{1}{4}$ of the CPU time required for MULNORS values.

In most cases, the TS value reasonably approximates the trivariate normal probability. The approximation is best when all of the correlations are 0.1; this is not surprising as the first order approximation (4) with $h(y)=1$ reduces to the exact marginal probabilities when all correlations are equal to zero. The TS method performs reasonably well in most of the other situations examined. The poorest approximation is noted when all of the correlations equal 0.9 and the lower bounds are also equal. (A similar result was found for bivariate normal probabilities; data are not shown.) If greater accuracy is required, more terms can be added to the TS approximation.

Upper tail probabilities were also computed using the first-order TS approximation for standard $n$-dimensional $(n=2, \ldots, 20)$ normal distributions in the case with correlations all equal to 0.5 and lower bounds all equal to 0.0 . The exact probability can be computed using the formula $1 /(n+1)$. The results (Table 2) indicate that the TS approximation is better at lower dimensions, but may be useful even at

Table 1

Approximation of trivariate normal probabilities ${ }^{\text {a }}$

\begin{tabular}{|c|c|c|c|c|c|c|}
\hline \multicolumn{3}{|c|}{ Lower bounds } & \multicolumn{3}{|c|}{$\rho_{12}=\rho_{13}=\rho_{23}$} & \multirow{2}{*}{$\begin{array}{l}\rho_{12}=\rho_{23}=0.3 \\
\rho_{13}=-0.3\end{array}$} \\
\hline$Y_{1}$ & $Y_{2}$ & $Y_{3}$ & 0.1 & 0.5 & 0.9 & \\
\hline \multirow[t]{2}{*}{-2.0} & -2.0 & -2.0 & 0.93431 & 0.94253 & 0.96170 & 0.93586 \\
\hline & & & 0.93553 & 0.96171 & 0.97725 & 0.95066 \\
\hline \multirow[t]{2}{*}{-1.0} & -1.0 & -1.0 & 0.61064 & 0.67778 & 0.77317 & 0.61912 \\
\hline & & & 0.61188 & 0.70843 & 0.83928 & 0.64584 \\
\hline \multirow[t]{2}{*}{0.0} & 0.0 & 0.0 & 0.14891 & 0.25000 & 0.39233 & 0.14924 \\
\hline & & & 0.14872 & 0.25420 & 0.46236 & 0.15316 \\
\hline \multirow[t]{2}{*}{1.0} & 1.0 & 1.0 & 0.00736 & 0.03380 & 0.09734 & 0.00614 \\
\hline & & & 0.00731 & 0.03248 & 0.11172 & 0.00588 \\
\hline \multirow[t]{2}{*}{2.0} & 2.0 & 2.0 & 0.00005 & 0.00137 & 0.01013 & 0.00002 \\
\hline & & & 0.00005 & 0.00126 & 0.01066 & 0.00002 \\
\hline \multirow[t]{2}{*}{-1.0} & 0.0 & 1.0 & 0.07917 & 0.12551 & 0.15795 & 0.08545 \\
\hline & & & 0.07922 & 0.12716 & 0.15853 & 0.08787 \\
\hline \multirow[t]{2}{*}{0.0} & 1.0 & 2.0 & 0.00324 & 0.01261 & 0.02550 & 0.00314 \\
\hline & & & 0.00323 & 0.01271 & 0.02265 & 0.00314 \\
\hline \multirow[t]{2}{*}{0.0} & 1.0 & 1.0 & 0.01917 & 0.05622 & 0.11543 & 0.02273 \\
\hline & & & 0.01912 & 0.05596 & 0.12750 & 0.02321 \\
\hline \multirow[t]{2}{*}{-1.0} & -1.0 & 0.0 & 0.37280 & 0.44377 & 0.49865 & 0.36407 \\
\hline & & & 0.37337 & 0.45463 & 0.49998 & 0.37686 \\
\hline \multirow[t]{2}{*}{-2.0} & 0.0 & 2.0 & 0.01338 & 0.02072 & 0.02275 & 0.01652 \\
\hline & & & 0.01338 & 0.02081 & 0.02275 & 0.01675 \\
\hline
\end{tabular}

\footnotetext{
a First entry is either table value (equal correlations and first five rows) or MULNORS value, second entry is Taylor series approximation.
} 
Table 2

Approximation of multivariate normal probabilities a

\begin{tabular}{lll}
\hline $\begin{array}{l}\text { Dimension } \\
\text { of integral }\end{array}$ & Exact value & $\begin{array}{l}\text { Taylor series } \\
\text { approximation }\end{array}$ \\
\hline 2 & 0.33333 & 0.33373 \\
3 & 0.25000 & 0.25420 \\
4 & 0.20000 & 0.20088 \\
5 & 0.16667 & 0.16381 \\
7 & 0.12500 & 0.11545 \\
10 & 0.09091 & 0.07438 \\
15 & 0.06250 & 0.04030 \\
20 & 0.04962 & 0.02373 \\
\hline
\end{tabular}

a Standard multivariate normal with correlations all equal to 0.5 and lower bounds all equal to 0.0 .

dimensions as high as 20. For correlations smaller than 0.5 , the approximation is expected to perform fairly well in this range of dimensions.

\subsection{The Mendell-Elston approximation to the multivariate normal}

The first-order TS approximation (4) is similar to the Mendell-Elston (1974) approximation to multivariate normal probabilities (see also Rice et al., 1979). In the bivariate case, the Mendell-Elston (ME) approximation can be derived as follows. Let $Y=\left(Y_{1}, Y_{2}\right)^{\mathrm{T}}$ be a bivariate normal random variable with joint density

$$
\phi(y)=(2 \pi)^{-1}(\operatorname{det} \Sigma)^{-1 / 2} \exp \left[-\frac{1}{2}(y-\mu)^{\mathrm{T}} \Sigma^{-1}(y-\mu)\right],
$$

where $\mu=\left(\mu_{1}, \mu_{2}\right)^{\mathrm{T}}$ is the mean vector and

$$
\Sigma=\left[\begin{array}{cc}
\sigma_{1}^{2} & \rho \sigma_{1} \sigma_{2} \\
\rho \sigma_{1} \sigma_{2} & \sigma_{2}^{2}
\end{array}\right]
$$

is the covariance matrix. Also let $R_{1} \times R_{2}=\left(h_{1}, \infty\right) \times\left(h_{2}, \infty\right)$ be the region of integration and denote $P\left(Y_{2} \geqslant h_{2}\right)$ by $S\left(h_{2}\right)$ and $\phi\left(h_{2}\right) / S\left(h_{2}\right)$ by $a_{2}$. Then the following standard results can be obtained:

$$
\begin{aligned}
& m_{2}=E\left[Y_{2} \mid Y_{2} \geqslant h_{2}\right]=\mu_{2}+\sigma_{2} a_{2}, \\
& m_{1 \mid 2}=E\left[Y_{1} \mid Y_{2} \geqslant h_{2}\right]=\mu_{1}+\left(m_{2}-\mu_{2}\right) \rho \sigma_{1} / \sigma_{2},
\end{aligned}
$$

and

$$
s_{1 \mid 2}^{2}=\operatorname{Var}\left[Y_{1} \mid Y_{2} \geqslant h_{2}\right]=\sigma_{1}^{2}\left(1-\rho^{2} a_{2}\left(a_{2}-z_{2}\right)\right),
$$

where $z_{2}=\left(h_{2}-\mu_{2}\right) / \sigma_{2}$.

Thus the mean and variance of $Y_{1}$, given $Y_{2} \geqslant h_{2}$, are obtained. The distribution of $Y_{1}$, given $Y_{2} \geqslant h_{2}$, is not in general normal, but is assumed to be approximately normal so that $P\left(Y_{1} \geqslant h_{1}, Y_{2} \geqslant h_{2}\right)$ can be approximated by $S\left(h_{2}\right) S\left(z_{1}\right)$, where $z_{1}=\left(h_{1}-m_{1 \mid 2}\right) / s_{1 \mid 2}$. The approximation has been found to work well, especially for small $\rho$.

Both the ME and first-order TS approximations can be written as the product of a marginal probability and a conditional probability. In both cases, the mean of the conditional density is $m_{1 \mid 2}$, the mean of $Y_{1}$, conditional on $Y_{2} \geqslant h_{2}$. The ME approximation differs from the first-order TS approximation in the variance used in the conditional distribution of $Y_{1}$ given $Y_{2}$. The ME approximation uses the variance $s_{1 / 2}^{2}$ computed assuming truncation of $Y_{2}$, while the TS approximation uses the untruncated conditional 
variance $\sigma_{1}^{2}-\rho^{2} \sigma_{1} / \sigma_{2}$, which does not depend on the value of $h_{2}$. Like the TS approximation, the ME approximation does not require a structured covariance matrix, reduces to the exact marginal probabilities when all of the correlations are equal to zero, and has a simple form. Unlike the TS approximation, it does not provide a means for improving accuracy.

\subsection{Bivariate normal probabilities using the Taylor series}

Bivariate normal probabilities can be approximated using the TS method by taking $h(y)=1$ and $f(y)$ equal to the bivariate normal density $\phi(y)$ as defined in Section 3.2. In addition, let $E_{2}$ denote the marginal expected value of $Y_{2}$, conditional on $Y_{2} \in R_{2}$, and let $V_{2}$ denote the second central (around $E_{2}$ ) moment of the marginal distribution of $Y_{2}$, conditional on $Y_{2} \in R_{2} . E_{2}$ and $V_{2}$ take the form of a ratio of univariate integrals; for example,

$$
V_{2}=\int_{R_{2}}\left(y_{2}-E_{2}\right)^{2} \phi\left(y_{2}\right) \mathrm{d} y_{2} / \int_{R_{2}} \phi\left(y_{2}\right) \mathrm{d} y_{2} .
$$

Putting $h(\boldsymbol{y})=1$, the terms of the Taylor series expansion up to and including the second moment can be written

$$
\begin{aligned}
& \left(\int_{R_{1}} \phi\left(y_{1} \mid E_{2}\right) \mathrm{d} y_{1}\right)\left(1-\frac{1}{2} \frac{\rho \sigma_{1} V_{2}}{\sigma_{2}}\left(\frac{\rho}{\sigma_{1} \sigma_{2}\left(1-\rho^{2}\right)}\right)\right) \\
& \quad+\frac{1}{2}\left(\int_{R_{1}}\left(y_{1}-\mu_{1}-\frac{\rho \sigma_{1}}{\sigma_{2}}\left(E_{2}-\mu_{2}\right)\right)^{2} \phi\left(y_{1} \mid E_{2}\right) \mathrm{d} y_{1}\right) V_{2}\left(\frac{\rho}{\sigma_{1} \sigma_{2}\left(1-\rho^{2}\right)}\right)^{2} .
\end{aligned}
$$

This expression is written in a form which groups the univariate integrals. Note that if the correlation is 0 , $\rho=0$ and the expression reduces to the exact marginal expectation. The approximation requires the evaluation of the univariate integrals in expression (5), the univariate integrals which represent $E_{2}, V_{2}$, and the marginal probability $P\left(Y_{2}>y_{2}\right)$.

\subsection{Comparison of Taylor series and Mendell-Elston methods}

If $h(y)=1$, expression (5), when multiplied by $\int_{R_{2}} \phi\left(y_{2}\right) \mathrm{d} y_{2}$, approximates bivariate normal probabilities. The adequacy of the TS and ME approximations for computing bivariate normal upper tail probabilities was examined by computing approximate values of the probability for values of the lower bounds ranging from -2.0 to 2.0 for a bivariate normal distribution with means of 0 , variances of 1 , and a correlation coefficient ranging from -0.5 to 0.5 . A subset of the results are shown in Table 3 , along with the exact values (U.S. National Bureau of Standards, 1959). The TS values were computed using terms up to and including the second moment of the Taylor series expansion (i.e., the first two terms of equation (5) with $h(y)=1$ ). The univariate integrals required for the approximation were computed by quadrature using the IMSL subroutine DMLIN.

The results in Table 3 suggest that both approximations are quite good when $|\rho|=0.1$. Both approximations are poorer but still adequate when $|\rho|=0.5$. The TS method generally provides better approximations than the ME method when $\rho=0.5$ and the joint tail probability is large; the ME method generally performs better than the TS method when $\rho=-0.5$ and the joint probability is large. When $\rho=-0.9$ (data not shown), the TS method is more accurate than the ME method when the joint tail probability is small and less accurate when the joint tail probability is large. When $\rho=0.9$ (data not shown), both methods are equally accurate on average, although both are less accurate than for small $|\rho|$.

For the TS approximation, a natural question arises regarding the ordering of the variables, i.e., the choice of variable on which to condition. If the marginal tail probability of $Y_{2}$, say, is small, $V_{2}$ and the 
Table 3

Approximation of bivariate normal probabilities ${ }^{\text {a }}$

\begin{tabular}{|c|c|c|c|c|c|}
\hline \multicolumn{2}{|c|}{ Lower bounds } & \multicolumn{4}{|l|}{$\rho$} \\
\hline$Y_{1}$ & $Y_{2}$ & -0.5 & -0.1 & 0.1 & 0.5 \\
\hline \multirow{3}{*}{-2.0} & -2.0 & 0.954503 & 0.954780 & 0.955372 & 0.958553 \\
\hline & & 0.956324 & 0.954786 & 0.955367 & 0.959154 \\
\hline & & 0.955069 & 0.954785 & 0.955367 & 0.957860 \\
\hline \multirow{3}{*}{-1.0} & -1.0 & 0.686472 & 0.702300 & 0.714009 & 0.745203 \\
\hline & & 0.684176 & 0.702297 & 0.714007 & 0.743404 \\
\hline & & 0.686222 & 0.702299 & 0.714009 & 0.744651 \\
\hline \multirow[t]{3}{*}{0.0} & 0.0 & 0.166667 & 0.234058 & 0.265942 & 0.333333 \\
\hline & & 0.166266 & 0.234050 & 0.265950 & 0.333734 \\
\hline & & 0.165880 & 0.234050 & 0.265950 & 0.334120 \\
\hline \multirow[t]{3}{*}{1.0} & 1.0 & 0.003782 & 0.019610 & 0.031320 & 0.062514 \\
\hline & & 0.003857 & 0.019611 & 0.031320 & 0.062744 \\
\hline & & 0.003866 & 0.019610 & 0.031320 & 0.062719 \\
\hline \multirow[t]{3}{*}{2.0} & 2.0 & 0.000003 & 0.000280 & 0.000872 & 0.004053 \\
\hline & & 0.000003 & 0.000280 & 0.000872 & 0.004059 \\
\hline & & 0.000004 & 0.000280 & 0.000872 & 0.004057 \\
\hline \multirow[t]{3}{*}{-2.0} & -1.0 & 0.818715 & 0.821028 & 0.823641 & 0.831861 \\
\hline & & 0.820255 & 0.821035 & 0.823635 & 0.831674 \\
\hline & & 0.819746 & 0.821035 & 0.823634 & 0.831073 \\
\hline \multirow[t]{3}{*}{-2.0} & 0.0 & 0.479276 & 0.486482 & 0.490769 & 0.497974 \\
\hline & & 0.479832 & 0.486485 & 0.490766 & 0.497877 \\
\hline & & 0.479798 & 0.486485 & 0.490766 & 0.497777 \\
\hline \multirow[t]{3}{*}{-2.0} & 1.0 & 0.145389 & 0.153609 & 0.156222 & 0.158508 \\
\hline & & 0.145433 & 0.153610 & 0.156221 & 0.158501 \\
\hline & & 0.145451 & 0.153610 & 0.156221 & 0.158496 \\
\hline \multirow[t]{3}{*}{-1.0} & 1.0 & 0.096141 & 0.127335 & 0.139045 & 0.154873 \\
\hline & & 0.095911 & 0.127335 & 0.139045 & 0.154798 \\
\hline & & 0.095936 & 0.127335 & 0.139045 & 0.154789 \\
\hline \multirow[t]{3}{*}{0.0} & 1.0 & 0.031257 & 0.069674 & 0.088981 & 0.127398 \\
\hline & & 0.031286 & 0.069674 & 0.088982 & 0.127369 \\
\hline & & 0.031241 & 0.069673 & 0.088982 & 0.127414 \\
\hline \multirow[t]{3}{*}{1.0} & 2.0 & 0.000147 & 0.002433 & 0.005046 & 0.013266 \\
\hline & & 0.000150 & 0.002433 & 0.005046 & 0.013279 \\
\hline & & 0.000150 & 0.002433 & 0.005046 & 0.013280 \\
\hline
\end{tabular}

${ }^{a}$ First entry is table value, second entry is Taylor series approximation and third entry is Mendell-Elston approximation.

higher moments are small and thus the terms in the Taylor expansion corresponding to the second and higher moments are small and the series converges rapidly, assuming that the series does in fact converge. Thus, a better approximation should be achieved by conditioning on the variable with the smaller marginal tail probability. In fact, if the marginal tail probability of $Y_{2}$ is very small, an excellent approximation can be achieved by using only $g\left(E_{2}\right)=\int_{R_{1}} \phi\left(y_{1} \mid E_{2}\right) \mathrm{d} y_{1}$. The results in Table 3 were obtained by conditioning on $Y_{2}$, because the marginal tail probability for $Y_{1}$ was always at least as great as that for $Y_{2}$. Results obtained by conditioning on $Y_{1}$ (not shown) were poorer than those shown in Table 3.

\section{Approximation of conditional expectations for censored data}

Consider the problem of parameter estimation for the bivariate normal distribution when some observations are censored. Censoring occurs in failure time data when only a lower bound on the failure time for an individual is available. For example, it may be known that an individual was still alive at the last 
observation time $y$, so that the outcome variable, time of death $T$, is known only to be greater than $y$. Because censored data is a special form of missing data, one approach (Pettitt, 1986; Olson and Weissfeld, 1990) to parameter estimation is to use the EM algorithm (Dempster, Laird and Rubin, 1977). This algorithm requires computation of the expected values of the sufficient statistics of the complete data likelihood, conditional on the data and the current parameter estimates. Specifically, the conditional expectations of $T_{1}, T_{2}, T_{1}^{2}, T_{2}^{2}$ and $T_{1} T_{2}$ are required for each observation. When only one observation in a pair is censored, these conditional expectations are ratios of univariate integrals which can be evaluated easily. When both observations in a pair are censored, the conditional expectations are ratios of bivariate integrals which can be approximated using the TS approach.

Let $\left(T_{1}, T_{2}\right)^{\mathrm{T}}$ be an observation from a bivariate normal distribution with joint density $\phi\left(t_{1}, t_{2}\right)$ and let $\left(C_{1}, C_{2}\right)^{\mathrm{T}}$ be a vector of censoring times with $C_{i}$ independent of $T_{i}, i=1,2$. Then $Y_{i}=\min \left(T_{i}, C_{i}\right)$ and $\delta_{i}=I\left(T_{i} \leqslant C_{i}\right), i=1,2$, represent the observed pair of data. If $\delta=\left(\delta_{1}, \delta_{2}\right)=(0,0)$, then both observations in the pair are censored and we know only that each failure time $T_{i}$ exceeds its observed (censoring) time $Y_{i}$. In this case, the required conditional expectations take the form

$$
E\left[h\left(T_{1}, T_{2}\right) \mid T_{1}>y_{1}, T_{2}>y_{2}, \mu, \Sigma\right]=\frac{\int_{y_{2}}^{\infty} \int_{y_{1}}^{\infty} h\left(t_{1}, t_{2}\right) \phi\left(t_{1}, t_{2}\right) \mathrm{d} t_{1} \mathrm{~d} t_{2}}{\int_{y_{2}}^{\infty} \int_{y_{1}}^{\infty} \phi\left(t_{1}, t_{2}\right) \mathrm{d} t_{1} \mathrm{~d} t_{2}} .
$$

Dividing the numerator and denominator of this equation by $\int_{y_{2}}^{\infty} \phi\left(t_{2}\right) \mathrm{d} t_{2}$ and rewriting as before, we get

$$
\begin{gathered}
\int_{y_{2}}^{\infty} \phi\left(t_{2}\right) \int_{y_{1}}^{\infty} h\left(t_{1}, t_{2}\right) \phi\left(t_{1} \mid t_{2}\right) \mathrm{d} t_{1} \mathrm{~d} t_{2} / \int_{y_{2}}^{\infty} \phi\left(t_{2}\right) \mathrm{d} t_{2} \\
\int_{y_{2}}^{\infty} \phi\left(t_{2}\right) \int_{y_{1}}^{\infty} \phi\left(t_{1} \mid t_{2}\right) \mathrm{d} t_{1} \mathrm{~d} t_{2} / \int_{y_{2}}^{\infty} \phi\left(t_{2}\right) \mathrm{d} t_{2} \\
=\frac{E_{T_{2}}\left(\int_{y_{1}}^{\infty} h\left(t_{1}, T_{2}\right) \phi\left(t_{1} \mid T_{2}\right) \mathrm{d} t_{1} \mid T_{2}>y_{2}\right)}{E_{T_{2}}\left(\int_{y_{1}}^{\infty} \phi\left(t_{1} \mid T_{2}\right) \mathrm{d} t \mid T_{2}>y_{2}\right)} .
\end{gathered}
$$

The numerator and the denominator can now be separately expanded about $E_{T_{2}}\left(T_{2} \mid T_{2}>y_{2}\right)$, the marginal expectation of $T_{2}$, conditional on the censored value $y_{2}$. The denominator is the same bivariate tail probability examined in Section 3 and can be approximated in the same manner. The numerator can be approximated by setting $h\left(t_{1}, t_{2}\right)$ equal to $t_{1}, t_{2}, t_{1}^{2}, t_{2}^{2}$ or $t_{1} t_{2}$ and performing the appropriate expansion. The choice of order (i.e., variable to be conditioned upon) affects the actual form of the expansion; as noted in Section 3, a better approximation is achieved by conditioning on the variable with the smallest marginal tail probability.

The adequacy of the TS approximation was examined using a standard bivariate normal distribution with $\rho=0.5$. The approximation was based on terms up to and including the third moment for both the numerator and denominator and computed for values of $y_{1} \leqslant y_{2}$ of $-2.0,-1.5,-1.0,-0.5,0.0,0.5,1.0$, 1.5 and 2.0. In all instances, approximate values were obtained by conditioning on $T_{2}$, the variable with the smallest marginal tail probability. The univariate integrals were again computed by quadrature using DMLIN. Comparison values were computed by bivariate quadrature also using DMLIN. A subset of the results are given in Table 4.

In general, the approximation is very accurate provided that censoring does not occur in the lower tails of the marginal distributions. Accuracy of the approximation of the second moments, particularly $E\left(T_{1} T_{2}\right)$, 
Table 4

Approximation of conditional expectations for censored bivariate normal data ${ }^{\text {a }}$

\begin{tabular}{|c|c|c|c|c|c|c|}
\hline \multicolumn{2}{|c|}{ Lower bounds } & \multicolumn{5}{|c|}{ Conditional expectations } \\
\hline$T_{1}$ & $T_{2}$ & $E\left(T_{1}\right)$ & $E\left(T_{2}\right)$ & $E\left(T_{1}^{2}\right)$ & $E\left(T_{2}^{\mathbf{2}}\right)$ & $E\left(T_{1} T_{2}\right)$ \\
\hline \multirow[t]{2}{*}{-2.0} & -2.0 & 0.07400 & 0.07400 & 0.88166 & 0.88166 & 0.41133 \\
\hline & & 0.07033 & 0.07033 & 0.89035 & 0.89035 & 0.42706 \\
\hline \multirow[t]{2}{*}{-1.0} & -1.0 & 0.34978 & 0.34978 & 0.75600 & 0.75600 & 0.36178 \\
\hline & & 0.35049 & 0.35049 & 0.75906 & 0.75906 & 0.37042 \\
\hline \multirow[t]{2}{*}{0.0} & 0.0 & 0.89762 & 0.89762 & 1.20676 & 1.20676 & 0.91351 \\
\hline & & 0.89979 & 0.89979 & 1.20993 & 1.20993 & 0.91872 \\
\hline \multirow[t]{2}{*}{1.0} & 1.0 & 1.63643 & 1.63643 & 2.92970 & 2.92970 & 2.72296 \\
\hline & & 1.63699 & 1.63699 & 2.93047 & 2.93047 & 2.73315 \\
\hline \multirow[t]{2}{*}{2.0} & 2.0 & 2.47991 & 2.47991 & 6.31470 & 6.31470 & 6.16955 \\
\hline & & 2.47958 & 2.47958 & 6.31225 & 6.31225 & 6.18713 \\
\hline \multirow[t]{2}{*}{-2.0} & -1.0 & 0.17184 & 0.29500 & 0.87662 & 0.71622 & 0.35059 \\
\hline & & 0.16996 & 0.29269 & 0.88057 & 0.72074 & 0.33540 \\
\hline \multirow[t]{2}{*}{-2.0} & 0.0 & 0.40984 & 0.79948 & 0.98271 & 1.00290 & 0.50578 \\
\hline & & 0.40924 & 0.79889 & 0.98374 & 1.00297 & 0.50666 \\
\hline \multirow[t]{2}{*}{-2.0} & 1.0 & 0.76536 & 1.52536 & 1.37787 & 2.52589 & 1.26642 \\
\hline & & 0.76524 & 1.52524 & 1.37802 & 2.52573 & 1.26650 \\
\hline \multirow[t]{2}{*}{-1.0} & 1.0 & 0.81372 & 1.52986 & 1.36953 & 2.54131 & 1.33660 \\
\hline & & 0.81372 & 1.52865 & 1.36980 & 2.54104 & 1.33785 \\
\hline \multirow[t]{2}{*}{0.0} & 1.0 & 1.07064 & 1.55832 & 1.61875 & 2.64174 & 1.73748 \\
\hline & & 1.07117 & 1.55711 & 1.61997 & 2.65005 & 1.74093 \\
\hline \multirow[t]{2}{*}{-2.0} & 2.0 & 1.18710 & 2.37324 & 2.18624 & 5.74656 & 2.87435 \\
\hline & & 1.18708 & 2.37322 & 2.18624 & 5.74651 & 2.87433 \\
\hline \multirow[t]{2}{*}{1.0} & 2.0 & 1.77681 & 2.41459 & 3.47988 & 5.96271 & 4.32070 \\
\hline & & 1.77716 & 2.41525 & 3.48089 & 5.97241 & 4.32533 \\
\hline
\end{tabular}

airst entry is DMLIN value, second entry is Taylor series approximation.

is poorer than the approximation of the first moments. These conditional expectations were also approximated using a modification of the ME approximation (substitution of $s_{2 \mid 1}^{2}$ into the first-order TS approximation); the results (not shown), although quite good, were generally poorer than the results shown in Table 4.

\section{Discussion}

A Taylor series approximation to multivariate integrals taken with respect to multivariate probability distributions has been proposed. These multivariate integrals may be multivariate probabilities or components of expectations taken with respect to the multivariate distribution. In the case of the normal distribution, the method provides good approximations, particularly when the correlation coefficient is small in absolute value, compares well with the Mendell-Elston approximation and provides a means of improving accuracy by the addition of terms. For some applications, however, the number of terms required for an adequate approximation may be cumbersome.

The approximation requires the evaluation of univariate integrals, which may themselves be approximated if further ease of computation is required. The general methodology developed in Section 2 can easily be applied to other multivariate distributions requiring numerical integration. Extension to higher dimensional integrals is straightforward using repeated application of the principle demonstrated in equation (4). However, much preparatory analytic work may be required if improvement of the first-order approximation is desired. Further work is required to examine the usefulness of the approximation for higher order integrals involving multivariate distributions. 


\section{Acknowledgements}

We thank the anonymous referee for helpful comments.

\section{References}

Dempster, A.P., N.M. Laird and D.B. Rubin (1977), Maximum likelihood from incomplete data via the EM algorithm (with discussion), J. Roy. Statis. Soc. Ser. A 148, 82-117.

Johnson, N.L. and S. Kotz (1972), Distributions in Statistics: Continuous Multivariate Distributions (Wiley, New York) 43-53, 93-103.

Kres, H. (1983), Statistical Tables for Multivariate Analysis (Springer, New York).

Mendell, N.R. and R.C. Elston (1974), Multifactorial qualitative traits: genetic analysis and prediction of recurrence risks, Biometrics 30, 41-47.

Olson, J.M. and L.A. Weissfeld (1990), Parameter estimation from multivariate distributions in the presence of censoring, in preparation.
Pettitt, A.N. (1986), Censored observations, repeated measures and mixed effects models: an approach using the EM algorithm and normal errors, Biometrika 73, 635-643.

Rice, J., T. Reich and C.R. Cloninger (1979), An approximation to the multivariate normal integral: its application to multifactorial qualitative traits, Biometrics 35, 451-459.

Schervish, M.J. (1984), Multivariate normal probabilities with error bound, Appl. Statist. 33, 81-94.

U.S. National Bureau of Standards (1959), Tables of the Bivariate Normal Distribution Function and Related Functions, Applied Mathematics Series, 50. 